Perception \& Psychophysics

1982, 31 (1), 97-99

\section{S-R compatibility effects: Do we need a new theory?}

\author{
STEPHEN J. LUPKER and ALBERT N. KATZ \\ University of Western Ontario \\ London, Ontario N6A 5C2, Canada
}

It has been known for many years now that reaction time (RT) to a stimulus is shorter when its relative spatial location corresponds to the relative location of the required response (e.g., Fitts and Seeger, 1953). This result, termed the stimulus-response (S-R) compatibility effect, has been explained by Simon (Simon, 1968; Simon \& Rudell, 1967) as arising from a natural tendency to respond toward a source of stimulation. Compatible S-R pairs benefit from this tendency, while for incompatible pairs the tendency must be suppressed before correct responding can occur.

Cotton, Tzeng, and Hardyck $(1977,1980)$ have recently reported a series of studies challenging this conceptualization. The task employed in a majority of these studies involved the subjects' responding to one of four lights forming the four corners of an imaginary square about a fixation point (see Figure 1). Two response keys were used, one mapped to the top two lights, the other to the bottom two lights. Responding was unimanual.

The manipulation crucial to Cotton et al.'s (1977, 1980) argument involved rotating the response keys $90 \mathrm{deg}$ from their standard position parallel to the plane of the subject's body so that they were in line with the subject's midsaggital plane. In this placement, the keys were symmetric with respect to the stimuli occurring to the left and right of fixation, and thus Cotton et al. claimed that no S-R compatibility effects should be anticipated. Nonetheless, a pattern of results was obtained in both Experiment 2 of Cotton et al. (1977) with a 1-sec stimulus duration and Experiment 1 of Cotton et al. (1980) with a 100msec stimulus duration that appeared to the authors to be very much like typical S-R compatibility effects. Reaction times were faster to the top light in the visual field ipsilateral to the hand of responding and to the bottom light in the visual field contralateral to the hand of responding. For example, when using the right hand, responses to Light 1 were faster than to Light 2, but responses to Light 3 were faster than to Light 4 . These results, as well as a replication

Preparation of this note was supported by Grants A6333 and A7040 from the Natural Sciences and Engineering Research Council of Canada. Requests for reprints should be sent to Stephen J. Lupker, Department of Psychology, University of Western Ontario, London, Ontario N6A 5C2, Canada.

\section{STIMULUS PATTERN
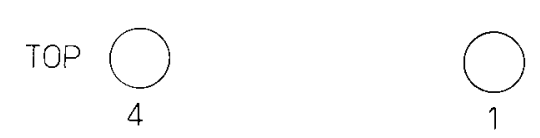

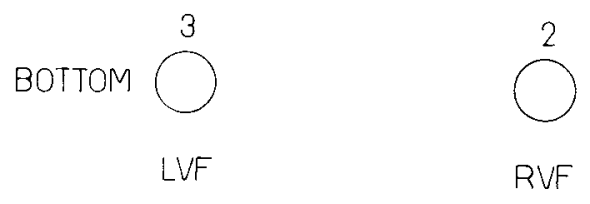

Figure 1. Stimulus display used by Cotton, Tzeng, and Hardyck (1977, 1980).

using more abstract stimuli to signal top and bottom, suggested to Cotton et al. (1980) that an alternate explanation of S-R compatibility effects might be in order.

What Cotton et al. offered was an input explanation based on the idea of functional differences in the two cerebral hemispheres. Their studies, as well as numerous others investigating S-R compatibility effects, involve stimuli presented in one visual field or the other and, thus, initially to one cerebral hemisphere or the other. The assumption is made that in the present studies the hand being used to respond produces a higher level of activation in the contralateral hemisphere (e.g., Kinsbourne, 1970). This hemisphere is then primed to process the most salient aspect of a stimulus, leaving the other hemisphere to process less salient aspects. With respect to the top-bottom (or above-below) comparison, an argument can be made that "above" is more salient than "below." As a result, top stimuli in the visual field contralateral to the activated hemisphere (and, therefore, ipsilateral to the hand of responding) should be processed faster than bottom stimuli in that same visual field. The opposite should occur in the visual field ipsilateral to the activated hemisphere, as was observed.

This theorizing, with its emphasis on input processing, represents a very new and different view of S-R compatibility effects. However, it is a view that finds little, if any, support in previous literature (see Katz, 1981) and, furthermore, is in fact inconsistent with the author's earlier data (Cotton et al., 1977, Experiment 1). In this earlier task, the four lights served as stimuli and the response keys were placed in their typical position parallel to the subject's body. Consider the situation in which the mapping is right key for the top lights and left key for the bottom lights. In this situation, RTs to right-field stimuli were observed to be faster for the top light, while 
RTs to left-field stimuli were faster for the bottom light, regardless of hand of responding. These results appear to be standard S-R compatibility effects and, as such, pose no problems for Simon's (1968) explanation of these effects. The authors' own theorizing, however, predicts these results only when the right hand is used for responding. When the left hand is used, the right hemisphere should be activated and, thus, the top light in the left field should be responded to more rapidly than the one below it. Similarly, the bottom light in the right field should enjoy an advantage over the light above it. These predictions are, of course, exactly opposite to the obtained results. Reversing the light-to-key mapping so that the right key is for the bottom lights and the left key is for the top lights reverses the problem. That is, although their predictions are now correct for left-hand responses, they are exactly opposite to the results obtained for right-hand responses. In essence, then, the authors' theorizing actually leads to the prediction that standard S-R compatibility effects should be reversed in certain situations, situations in which they themselves were not able to observe such a reversal.

Although Cotton et al.'s (1980) view of S-R compatibility effects can be rejected on the basis of their own data, the problem still remains of explaining the "compatibility" effects obtained in their other experiments. Is it necessary to postulate additional principles to explain the data obtained when the response keys are rotated $90 \mathrm{deg}$, as Cotton et al. (1980) claim? It appears one would need to argue in the affirmative only if it could be shown that no differential tendencies to respond toward the source of stimulation remained after rotating the response keys. This is ultimately an empirical question and certainly not an assumption one can simply accept. In fact, a survey of the available literature does suggest that the assumption is probably incorrect (see Annett \& Sheridan, 1973; Sheridan, 1973). Furthermore, an analysis of the motor movements in Cotton et al.'s $(1977,1980)$ tasks makes clear the nature of their stimulus-source response bias. When the response keys are placed in line with the midsagittal plane, the arm is presumably held essentially parallel to the plane of the body, and responding involves moving the hand toward or away from the body. When the right hand is held in that position, it seems likely that the most natural movement would be to pivot around the elbow or midforearm. This rotation creates a slight rightward movement when the hand is moved away from the body and a slight leftward movement when the hand is moved toward the body. Thus, any tendency to respond toward the source of stimulation would favor the upper right and lower left sources. With the left hand, the complete opposite would be expected (i.e., the most natural movements would be to pivot away and to the left or toward and to the right). In this case, any tendency to respond would favor the upper left and lower right sources of stimulation. If these tendencies were, in fact, operative, then Cotton et al.'s (1980) results would follow directly from Simon's (1968) explanation of S-R compatibility effects.

Cotton et al., while apparently aware of this alternate interpretation of their data, do not seem to be totally aware of its implications. They argue that if such tendencies were manifest in their task, subjects would actually be moving their hands slightly toward the source of stimulation and, thus, would be pressing the response keys on one edge or the other. In a further experiment, they reduced the diameter of the response keys so that hand movements were, of necessity, directly along the midsagittal plane. When this manipulation failed to alter the typical pattern of results, they argued that even this modified version of Simon's hypothesis could be ruled out.

The crucial point the authors appear to be missing is that, according to Simon's hypothesis, it is not necessary that an actual movement be made in the direction of the source of stimulation for S-R compatibility effects to arise. In fact, if such were the case, the hypothesis could be rejected essentially out of hand. For example, consider the simple experimental situation in which the stimuli are two lights, one to each side of fixation, with a response button under each. If subjects are required to place the index finger of each hand on the ipsilateral button, a situation is created in which no movement toward either stimulus is required. Thus, RT should not vary as a function of the light-to-key mapping. Yet, as Anzola, Bertoloni, Buchtel, and Rizzolatti (1977) and numerous others have shown, subjects' performance is much better when the lights are mapped to the keys directly below them than vice versa. What is relevant here, as well as in Cotton et al.'s $(1977,1980)$ studies, is not the actual movement but the entire set of components making up the response. A natural association between a stimulus and any of these components, from the selection of the response code (Wallace, 1971) to the actual movement of the appendage, can produce a tendency to respond to that stimulus. In Cotton et al.'s studies, the natural association between the two forms of movement (i.e., away and right or toward and left for right-handed responders) quite likely acts to create just such a tendency, probably by influencing the ease of selecting the response code. Thus, these movements should be executed more rapidly, while movements in the opposite direction should suffer, just as Cotton et al. observed. As such, unless more compelling data can be produced, it seems much more reasonable to retain the standard S-R compatibility explanation than to invoke one based on questionable hemisphere assumptions and supported by logically inconsistent data. 


\section{REFERENCES}

Annett, J., \& Sheridan, M. The effects of S-R and R-R compatibility on bimanual movement. Quarterly Journal of Experimental Psychology, 1973, 25, 247-252.

Anzola, G. P., Bertoloni, G., Buchtel, H. A., \& Rizzolatti, G. Spatial compatibility and anatomical factors in simple and choice reaction time. Neuropsychologia, 1977, 15, 295-302.

Cotton, B., Tzena, O. J. L., \& Hardyck, C. A response instruction by visual field interaction: S-R compatibility effect or ?. Bulletin of the Psychonomic Society, 1977, 10, 475-477.

Cotron, B., Tzena, O. J. L., \& Hardyck, C. Role of cerebral hemispheric processing in the visual half-field stimulus-response compatibility effect. Journal of Experimental Psychology: Human Perception and Performance, 1980, 6, 13-23.

FitTs, P. M., \& Segaer, C. M. S-R compatibility: Spatial characteristics of stimulus and response codes. Journal of Experimental Psychology, 1953, 46, 199-210.

KATZ, A. N. Spatial compatibility effects with hemifield presenta- tion in a unimanual two-finger task. Canadian Journal of Psychology, 1981, 35, 63-68.

Kinseourne, M. The cerebral basis of lateral asymmetries in attention. Acta Psychologica, 1970, 33, 193-201.

Sheridan, M. Effects of S-R compatibility and task difficulty on unimanual movement time. Journal of Motor Behavior, 1973, $5,199.205$.

Simon, J. R. Effect of ear stimulation on reaction time and movement time. Journal of Experimental Psychology, 1968, 78, 344-346.

Simon, J. R., \& Rudelu, A. P. Auditory S-R compatibility: The effect of an irrelevant cue on information processing. Journal of Applied Psychology, 1967, 51, 300-304.

W Allace, R. J. S-R compatibility and the idea of a response code. Journal of Experimental Psychology, 1971, 88, 354-360.

(Manuscript received September 30, 1981; accepted for publication October 6, 1981.) 